> Les systèmes nerveux et immunitaire ont en commun de constituer dans l'organisme des réseaux complexes de cellules en interaction. Dans ces réseaux transitent en permanence d'énormes quantités d'informations, codées de façon très différente dans les deux cas. Ces informations doivent en particulier circuler de cellule en cellule. Dans le système nerveux, ce transfert a lieu au niveau des synapses. Récemment, les immunologistes ont adopté le terme de synapse immunologique pour désigner l'interface entre deux cellules du système immunitaire. Mais est-il légitime d'appeler cette structure synapse? Peut-on y mettre en évidence un mode d'organisation ou de fonctionnement en rapport avec ceux des synapses nerveuses? <

\section{Synapses \\ immunologiques \\ et synapses \\ neuronales}

\author{
Alain Trautmann, Patrick Revy, \\ Emmanuel Donnadieu, Georges Bismuth
}

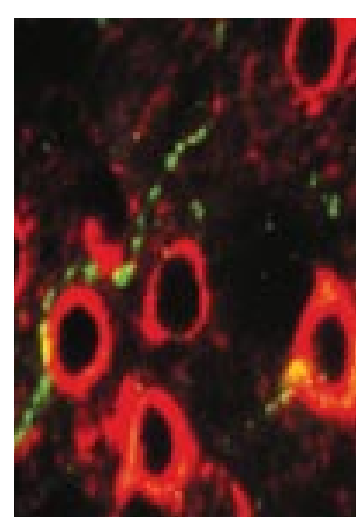

une cellule B pour provoquer sa différenciation, dans le cas d'une cellule T CD4 activée, etc. La synapse immunologique la plus étudiée est celle formée par une cellule T et une APC [2-4]. L'objet de cet article est d'examiner ces synapses T en utilisant comme élément de comparaison les synapses du système nerveux (synapses $\mathrm{N}$ ).

\section{Caractéristiques de la synapse T}

La structure moléculaire détectée par un lymphocyte T sur une APC n'est jamais un antigène soluble mais est constituée par l'association d'un peptide antigénique (du moins dans le cas des cellules T $\alpha \beta$ ) avec une molécule du complexe majeur d'histocompatibilité (CMH) de classe l ou de classe II. Dans cette reconnaissance, le récepteur de l'antigène ( $T$ cell receptor ou TCR) joue un rôle essentiel comme support de la spécificité, mais il fonctionne au sein d'un important complexe multimoléculaire «synaptique », différent selon les systèmes expérimentaux considérés. 


\section{Variété structurale des synapses T}

Plusieurs groupes ont montré que des «succédanés 》 d'APC (hybridomes B ou fibroblastes transfectés avec des $\mathrm{CMH}$ ) sont capables, à condition de présenter l'antigène approprié, de provoquer, en 10 à 30 minutes, la formation de complexes qui s'accumulent au centre de la synapse formant un CSMAC (central supramolecular activation cluster) entouré d'un PSMAC (peripheral supramolecular activation cluster) [5], voire d'un DSMAC (distal supramolecular activation cluster) [6] Le CSMAC comprend le $T C R$, le complexe CD3 qui lui est associé, et un ensemble de molécules de co-stimulation et de signalisation $[7,8]$. Le PSMAC comprend principalement des intégrines et des adaptateurs cytosquelettiques associés. Tous les types de molécules de surface ne s'accumulent pas à cette interface. Certaines, comme CD43, qui fonctionne sans doute comme une molécule répulsive, en sont au contraire exclues $[2,9,10]$.

$(\rightarrow) \mathrm{m} / \mathrm{s}$ $1999, n^{\circ} 8-9$, p. 931

$(\rightarrow) \mathrm{m} / \mathrm{s}$ 2003, $n^{\circ} 1$, p. 43
L'utilisation de protéines couplées à la GFP (green fluorescent protein) a permis d'observer le «ballet moléculaire » de CD3 et de CD4 vers la synapse ou hors de la synapse de la cellule T [11], et de ICAM-1 (intercellular adhesion molecule-1) sur les cellules d'un lymphome B utilisées comme APC [12]. Des mouvements moléculaires ont pu être observés pour une synapse $T$ très particulière dans laquelle l'APC n'est pas une cellule mais une bicouche lipidique plane contenant des molécules rendues fluorescentes par couplage chimique et ancrées dans la bicouche par des motifs GPI (glycophosphatidylinositol) [1]. Quelques minutes après l'arrivée des cellules T sur la bicouche, les «synapses » formées présentent des arrangements moléculaires très similaires aux SMAC, bien qu'ayant une topologie inverse dans les premières minutes du contact, le TCR entourant les molécules d'adhérence.

La similitude des résultats obtenus avec deux synapses artificielles ( $T$-hybridome $B$ et T-bicouche lipidique) a fait émerger la notion d'une synapse immunologique unique, qui passerait par plusieurs stades: une synapse immature initiale, avec des TCR périphériques, et la synapse «mature», avec une agrégation centrale des TCR. Cette synapse mature est souvent considérée comme le prototype de la synapse immunologique. Ce point de vue nous paraît incorrect pour deux raisons. D'abord, dans les cas où une synapse mature est formée, la structure qui la précède est aussi une synapse, c'està-dire une structure d'échange d'informations entre les deux cellules. Ensuite, nombre de synapses adoptent de préférence une structure multifocale et non pas concentrique. C'est le cas de la synapse formée entre thymocytes et cellules épithéliales thymiques [13], ou celui de la synapse T-DC [9], deux synapses d'importance phy- siologique majeure. Même pour la synapse T-lymphome $B$, la structure concentrique n'est vue qu'à forte concentration d'antigène, tandis qu'à plus basse concentration, une situation plus physiologique, le TCR/CD3 se concentre en foyers multiples [14]. Cette variété d'organisations va à l'encontre de l'idée d'une synapse prototypique, correspondant à un plan préétabli. Elle est en faveur de l'hypothèse selon laquelle la structure finale serait le résultat de phénomènes d'auto-assemblage entre molécules diffusant dans un réseau de contraintes complexes jusqu'à être piégées et immobilisées. Ces principes peuvent évoquer une forme de darwinisme à l'échelle moléculaire [15].

\section{La synapse T-DC}

Les cellules dendritiques (DC) sont les seules APC capables d'activer les lymphocytes T naïfs in vivo, d'où leur importance cruciale dans le déclenchement d'une réponse immunitaire $(\rightarrow)$. À l'état immature, les DC captent très efficacement les antigènes en périphérie, dans la peau et les muqueuses en particulier. Sous l'effet d'une lésion ou d'un autre signal inflammatoire, elles migrent vers les ganglions lymphatiques traversés par les lymphocytes $T$ naïfs où elles évoluent vers un phénotype dit mature, caractérisé notamment par une expression accrue de molécules du CMH et de co-stimulation leur conférant une efficacité exceptionnelle de présentation de l'antigène [16]. L'ensemble de ces propriétés particulières a fait utiliser les cellules dentritiques dans des essais cliniques d'immunothérapie des cancers $(\rightarrow)$.

Les contacts T-DC présentent la particularité de pouvoir se mettre en place en l'absence d'antigène et de CMH à la surface des $D C$, entraînant des réponses calciques dans les cellules $T$, avec augmentation locale des phosphotyrosines $[9,17,18]$. Ces résultats sont cohérents avec l'observation selon laquelle les $D C$ sont «scannés » par les lymphocytes T de façon indépendante de l'antigène [17, 19], et provoquent une mobilité accrue des cellules T qui pourrait faciliter la reconnaissance d'antigènes exogènes faiblement présentés. Récemment, l'observation par microscopie biphotonique in vivo d'interactions T-DC dans des organes lymphoïdes intacts a confirmé ces résultats $[20,21]$. Ces interactions ont sans doute aussi un rôle déterminant dans la survie des lymphocytes T naïfs [9] et dans le phénomène de préactivation ou priming, c'est-à-dire d'abaissement du seuil d'activation des lymphocytes T. Les tenants de la synapse Tà organisation concentrique prédisent que les membranes des deux cellules devraient être distantes d'environ $15 \mathrm{~nm}$ au niveau du cSMAC et de $40 \mathrm{~nm}$ au niveau du PSMAC. Cela permettrait à des molécules de 7-8 nm (comme le TCR et le CMH) d'interagir, repoussant les molécules de grande taille 
dans le PSMAC, voire en dehors de la synapse. Nos clichés de synapses T-DC en microscopie électronique (Figure 1) montrent en fait une fente synaptique avec une succession de distances pré- et post-synaptiques intercalées de $15 \mathrm{~nm}$ et de plusieurs dizaines de nm. Aucun espace synaptique prolongé d'environ $40 \mathrm{~nm}$ (prédit pour le pSMAC) n'est observé dans cette synapse, la seule analysée en microscopie électronique à ce jour.

\section{Variété fonctionnelle des synapses immunologiques}

À quoi servent les synapses immunologiques, et sont-elles importantes dans le déclenchement de la réponse à l'antigène? Dans une publication récente, le groupe de Shaw [22] a montré qu'au niveau de la synapse T-macrophage, les premiers signaux dépendants du TCR précèdent la formation de la synapse mature. Ils en concluent que la synapse immunologique ne serait pas impliquée dans la signalisation du TCR. On peut cependant leur objecter que
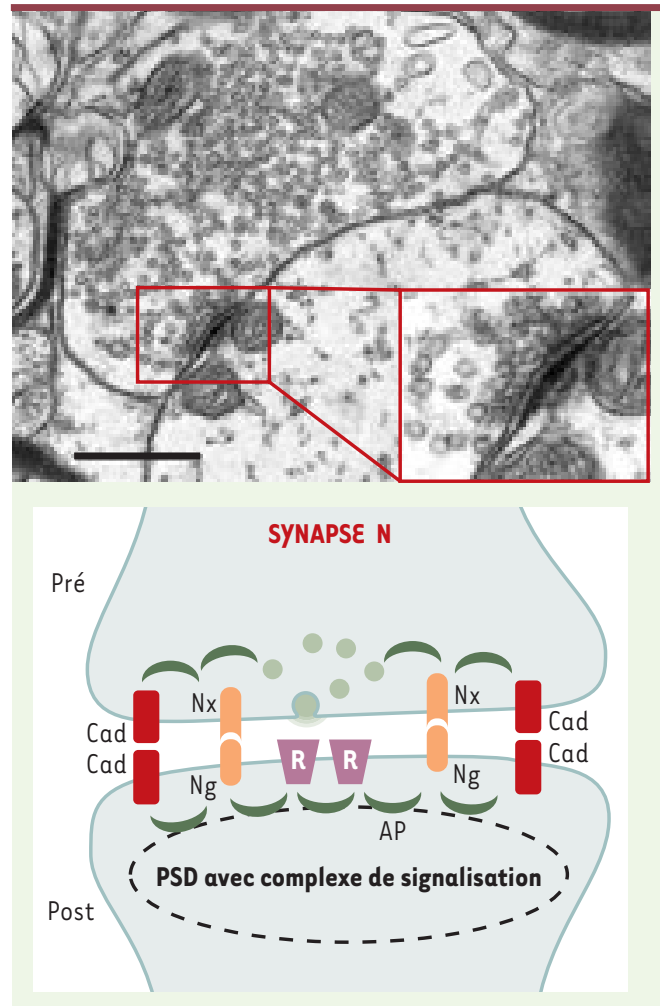

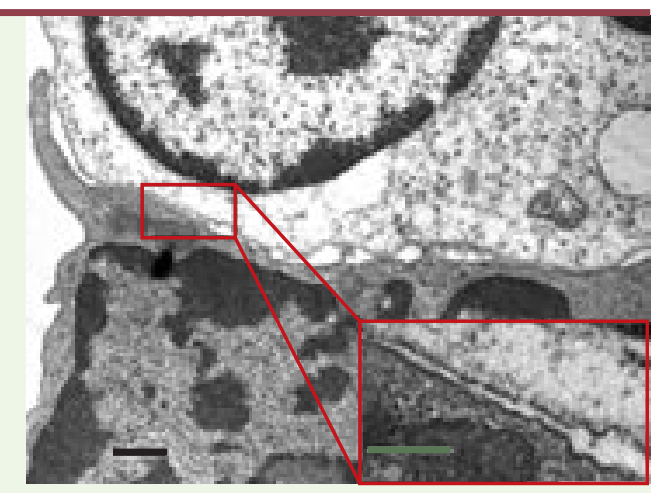

SYNAPSE T

Cellule dendritique

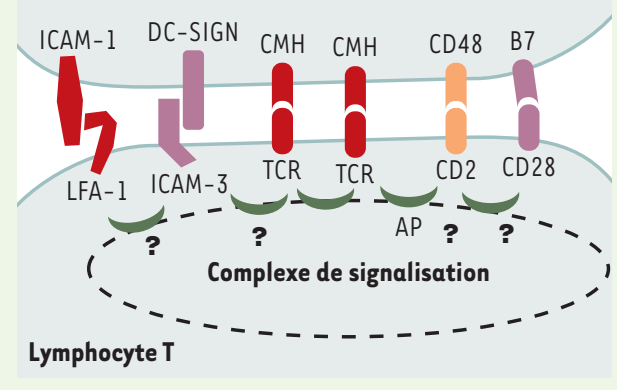

Figure 1. Comparaison des synapses du système nerveux et immunologiques (lymphocyte T). En haut: à gauche, cliché de microscopie électronique d'une synapse glycinergique (avec la permission du Dr A. Triller) et, à droite, d'une synapse T-cellule dendritique (avec la permission du Dr G. Raposo). Échelle: 0,5 $\mu \mathrm{m}$ ( $0,25 \mu \mathrm{m}$ pour l'encadré). La présence de «densités post-synaptiques » (PSD) est bien visible dans l'encadré de la synapse N. $\varepsilon_{n}$ bas: représentation schématique des molécules responsables de la formation des synapses $N$ (à gauche) et $T$ (à droite). R: récepteur de neurotransmetteur; Nx: neurexine; $\mathrm{Ng}$ : neuroligine; Cad: cadhérine; AP: adaptateurs primaires; PSD : densité post-synaptique; TCR: récepteur de l'antigène; CMH : complexe majeur d'histocompatibilité; ICAM-1: intercellular adhesion molecule 1; LFA-1: leukocyte function-associated antigen 1; DC-SIGN: dendritic cell-specific ICAM-3-grabbing non integrin. la synapse existe avant la structure finale, figée, de la synapse dite mature, et que plusieurs types de synapses 'adoptent que rarement la structure concentrique de la synapse mature. tout lieu de croire qu'elle permet ensuite la détection d'un nombre incroyablement faible de complexes $\mathrm{CMH}$-peptide, indique au contraire que cette synapse T-DC est fondamentale pour La formation d'un complexe multimoléculaire comprenant des molécules d'adhérence et de signalisation et des protéines du cytosquelette stabiliserait à la fois les contacts T-APC mais aussi l'induction de signaux comme ceux délivrés à arnapse par la PI3-kinase (phospho-inositide-3-kinase) [25, 26]. Cette activation maintenue provoquerait la prolifération du lymphocyte T.

Une autre fonction de la synapse pourrait être de favoriser la sécrétion polarisée de la cellule T. Cela peut s'appliquer à la sécrétion très rapide de molécules cytolytiques par les cellules T CD8 interagissant avec leur cible [27], ou à celle de cytokines par des T CD4 après plusieurs heures de contact avec une cellule $B$ [28], qui aura dans ce cas fonctionné successivement comme APC puis comme bénéficiaire, en retour, des cytokines $T$. Dans un cas comme dans l'autre, cette sécrétion est « signée » par la rotation du centrosome $T$ vers la cible ou la cellule $B$.

\section{Et la synapse neuronale?}

Nous allons voir maintenant en quoi une comparaison des synapses $T$ et des synapses du système nerveux (synapses $N$ ) peut être éclairante pour l'analyse des premières. Pas- 
sons rapidement sur une différence évidente: dans le système nerveux, la grande vitesse de circulation de l'information est rendue possible par son codage électrique, qui n'a pas d'équivalent dans le système immunitaire, dans lequel les cellules sont «non excitables » car dépourvues de canaux $\mathrm{Na}^{+}$ou $\mathrm{Ca}^{2+}$ activables par dépolarisation. Dans le système immunitaire, ce sont des cellules et des messagers solubles qui circulent. À un autre niveau de comparaison, peut-on considérer que le transfert d'information aux synapses $\mathrm{N}$ se fait essentiellement par des messagers solubles, les neurotransmetteurs, tandis que le transfert d'information aux synapses T passerait uniquement par des interactions directes entre protéines des membranes pré- et post-synaptiques? La situation n'est pas si simple.

Il est vrai qu'il n'y a pas de contact direct entre les cellules pré- et post-synaptiques aux jonctions neuro-musculaires des vertébrés. Mais les jonctions neuro-musculaires sont des synapses particulières et atypiques: la distance séparant les deux cellules est supérieure à 40 $\mathrm{nm}$, et une lame basale est interposée entre elles. Or, dans le système nerveux central, les synapses $\mathrm{N}$ ne comportent pas de lame basale, les fentes synaptiques sont étroites (15 à $30 \mathrm{~nm}$ ), et les deux cellules sont en contact direct par des interactions homophiliques (mêmes molécules) ou hétérophiliques (molécules différentes).

Les interactions homophiliques passent par les cadhé- $(\rightarrow) \mathrm{m} / \mathrm{s}$ 2000, n' 1 , p. 116 et 128 rines $(\rightarrow)$. Diverses cadhérines ont des profils d'expression spécifiques dans le cerveau, délimitant différentes «voies neurales» [29]. L'adhérence pré-post-synaptique dépendante de ces cadhérines est dynamique, localement contrôlée et modulée par l'activité synaptique. Les interactions hétérophiliques, quant à elles, mettent en jeu en particulier les $\beta$-neurexines pré-synaptiques et les neuroligines post-synaptiques [30]. Toutes deux forment des familles de protéines très polymorphes résultant de l'épissage alternatif de plusieurs gènes.

D'autres molécules pré- et post-synaptiques contrôlent les étapes initiales de la formation de la synapse $\mathrm{N}$, comme par exemple les récepteurs $\varepsilon p h \mathrm{~B}$ à activité tyrosine kinase et leurs ligands, les éphrines. De façon surprenante, certaines molécules fondamentales pour le fonctionnement du système immunitaire et en particulier de la synapse $T$, comme le $\mathrm{CMH}$ de classe I et $\mathrm{CD} 3 \zeta$, sont exprimées dans certains neurones, où leur absence provoque des anomalies du développement de certaines connexions neuronales [31].

\section{Les immunotransmetteurs existent-ils?}

Dans les synapses $\mathrm{N}$, à l'exception des jonctions neuromusculaires, l'étroitesse de la fente synaptique permet à la fois des interactions cellulaires directes et l'échange d'information via des molécules solubles. L'importance des interactions cellulaires directes a bien été établie dans les synapses $T$; en revanche, l'importance des facteurs solubles n'y a pas encore été étudiée en détail. Plus précisément, on sait qu'après plusieurs dizaines de minutes de contact entre un lymphocyte $T$ et un lymphocyte $B$ ou une cellule cible, la cellule T sécrète des cytokines ou des granules cytolytiques de façon polarisée vers la cellule $B$ ou la cellule cible [27, 28, 32]. Mais nous ignorons dans quelle mesure des facteurs solubles qu'on pourrait appeler immunotransmetteurs contribuent à la formation et au fonctionnement initial de la synapse $T$. Ce pourrait être le cas des chimiokines qui jouent un rôle crucial dans le recrutement des lymphocytes $T$ aux sites d'inflammation et qui contribuent aux étapes précoces d'adhérence des lymphocytes $T$ aux cellules endothéliales. Des données récentes obtenues au laboratoire ont mis en évidence la participation de chimiokines libérées par les DC dans l'interaction T-DC. On peut aussi envisager que des cytokines comme l'interleukine-2 puissent fonctionner comme immunotransmetteurs, dans la mesure où l'interleukine-2 induit une activité intense de formation-rétraction de replis membranaires (lamellipodes) [33] et potentialise la signalisation via le TCR [34].

\section{Regroupement de récepteurs et} adaptateurs spécifiques

Pour qu'une structure synaptique se mette en place et perdure, des mécanismes de recrutement et de concentration de récepteurs à cette synapse sont nécessaires. Les mécanismes présidant à ces phénomènes sont bien mieux connus pour les synapses $\mathrm{N}$ que pour les synapses $\mathrm{T}$.

\section{Synapses N}

Dans la terminaison nerveuse pré-synaptique, la forte densité et l'ouverture synchrone de canaux calciques permet de créer des microdomaines locaux et transitoires, à très forte concentration calcique intracellulaire, nécessaires à l'exocytose de vésicules contenant des neurotransmetteurs. Dans ces mêmes microdomaines, tous les éléments nécessaires à la fusion et au recyclage des vésicules synaptiques doivent être présents. Ces regroupements moléculaires dépendent notamment de la présence de protéines adaptatrices qui possèdent fréquemment des domaines PDZ (voir Encadré) et que l'on appellera « adaptateurs primaires » par opposition aux « adaptateurs secondaires » qui permettent notamment l'ancrage au cytosquelette. Parmi ces adaptateurs primaires, citons PSD95 pour les récepteurs NMDA ( $\mathrm{N}$-méthyl-D-aspartate) du glutamate, la géphyrine qui contribue à la formation 
de complexes de récepteurs de la glycine $[35,36]$ et la rapsyne pour les récepteurs de l'acétylcholine au niveau des jonctions neuro-musculaires [37]. Dans ce dernier cas, un mécanisme d'agrégation très particulier des récepteurs de l'acétylcholine est assuré par l'agrine présente dans la lame basale (voir Encadré).

Ainsi, la spécificité de la synapse $\mathrm{N}$ ne dépend pas seulement des interactions spécifiques des récepteurs post-synaptiques avec les neurotransmetteurs appropriés libérés par les terminaisons nerveuses, mais aussi de protéines - les neurexines et les neuroligines - qui contrôlent l'adhérence cellulaire et s'associent plus ou moins spécifiquement à ces protéines adaptatrices.

L'accumulation de récepteurs précède-t-elle ou suitelle la formation de la synapse? Probablement les deux. Les récepteurs NMDA peuvent initialement s'agréger en l'absence de PSD-95, mais leur association avec PSD-95 permet de maintenir le regroupement préformé de récepteurs à la synapse [38]. Le même type de relation semble s'établir entre les récepteurs de la glycine et la géphyrine [36].

\section{Synapses T}

Comment s'opère le regroupement de récepteurs à la synapse T? Curieusement, on ignore à peu près tout des adaptateurs primaires qui permettraient, d'une part, de regrouper les TCR et, d'autre part, d'associer les TCR aux molécules d'adhérence, à quelques exceptions près. L'une d'elles est CD2AP [39], qui interagit avec la molécule transmembranaire CD2 et permet son agrégation; une autre est ADAP/Fyb/SLAP130, qui couple l'activation du TCR à celle des intégrines et permet d'augmenter l'adhérence lors des contacts entre le lymphocyte $T$ et les cellules présentatrices d'antigènes $[40,41]$. Cette fonction pourrait également être assurée indirectement par des composants du complexe de signalisation déjà décrits. Les adaptateurs connus de la synapse T sont en fait pour la plupart des adaptateurs secondaires (voir Encadré). Les lymphocytes B et T expriment une protéine à domaine PDZ, hDLG (discs large homolog), qui est recrutée avec CD2 lors d'un regroupement de ces molécules induit par des anticorps anti-CD2 [42]. Mais, nous rappellerons qu'une agrégation moléculaire induite par un anticorps ne peut être considérée comme une synapse. Une autre molécule, EBP50, exprimée dans les lymphocytes $T$ et possédant deux domaines PDZ, pourrait faire le lien entre la membrane plasmique et le cytosquelette et régler négativement la formation de la synapse T [43]. Enfin, il faut mentionner la participation des protéines ERM (ezrine, radixine, moésine) (voir Encadré) qui jouent un rôle clé dans la construction de la synapse T en permettant l'exclusion de CD43.

\section{Modifications des synapses induites} par leur fonctionnement

Les synapses $\mathrm{N}$ sont des structures très dynamiques au cours du développement et ne peuvent être considérées comme des structures stables. Les enregistrements vidéo de neurones vivants exprimant de l'actine couplée à la GFP ont révélé des changements spontanés, rapides (à l'échelle de quelques secondes) et apparemment aléatoires de la forme des épines dendritiques, sortes de bourgeons où s'établissent les contacts synaptiques, qui contrastent avec la relative stabilité morphologique $\mathrm{du}$ «tronc » du dendrite [44]. Par ailleurs, il existe une relation réciproque entre la structure de la synapse et son fonctionnement. Au cours du développement d'une synapse, il faut qu'une forme rudimentaire de cette dernière se forme avant que des informations spécifiques de son (ou de ses) neurotransmetteur(s) ne puissent passer. Par la suite, l'activité synaptique affectera l'organisation de la synapse, et notamment la densité des récepteurs. Par exemple, la paralysie des neurones moteurs entraîne en quelques jours une baisse du nombre de récepteurs de l'acétylcholine présents à la synapse et leur redistribution à la surface du muscle [45]; au contraire, un blocage chronique des récepteurs NMDA dans les neurones de I'hippocampe augmente le regroupement synaptique de ces récepteurs [38]. De façon plus subtile, 30 minutes après la stimulation d'un neurone selon un profil particulier, des réorganisations de la synapse peuvent être observées [46].

Les mécanismes qui régissent les changements de structure de la synapse en fonction de l'activité synaptique ne sont pas élucidés mais il est possible que les adaptateurs primaires décrits plus haut soient impliqués. Ainsi, dans les synapses de drosophile, la distribution post-synaptique et la fonction de la protéine Discs large à domaine PDZ sont réglées par la protéine kinase CaMKII, dépendante du calcium et de la calmoduline [47].

Sur la base des observations que nous avons effectuées au niveau de la synapse T-DC, et contrairement à la théorie dominante de «l'antigène d'abord », il nous paraît probable qu'à l'instar de la synapse $N$, la formation d'une synapse $T$ rudimentaire précède la reconnaissance de l'antigène, après quoi les signaux spécifiques liés à l'antigène modifieraient sa structure et son fonctionnement. Au niveau de la synapse T-DC, tout se passe comme si la $D C$ émettait une «double injonction » contradictoire, d'une part en délivrant des signaux stimulant vigoureusement la déformation de la membrane du lymphocyte T et, d'autre part, en provoquant une augmentation de la concentration calcique intracellulaire qui tend à arrondir et à immobiliser le lymphocyte T [18]. Cette situation peut être comparée à celle des synapses glutamater- 
giques, où le glutamate pourrait d'abord induire le développement d'extensions membranaires (filopodes) mobiles par la stimulation de récepteurs NMDA ( $\alpha$ amino-3-hydroxy-5-methyl-4-isoxazole proprionate), puis, après l'apparition de récepteurs de type AMPA, déclencher un mécanisme qui réduirait la plasticité des épines dendritiques [48]. Des mécanismes antérogrades doivent aussi être pris en compte. Ainsi, lors des interactions T-DC, des réponses calciques [18] et des polarisations dynamiques d'actine dans les DC [49] ont été observées, suivies de l'envoi, par les DC, de chapelets de vésicules chargées en CMH de classe II [50]. Les DC reçoivent donc, des lymphocytes $T$, des signaux précoces qui peuvent influencer la formation de la synapse T-DC.

\section{Conclusions}

Le terme de «synapses immunologiques » est-il approprié pour décrire les interactions entre les cellules du système immunitaire? Oui, dans la mesure où les échanges d'informations entre cellules du système immunitaire ont lieu au niveau d'interfaces cellule-cellule qui évoquent les interfaces synaptiques $d u$ système nerveux. L'établissement d'un parallèle entre synapses $N$ et synapses T peut fournir de fructueux éléments de réflexion sur les deux systèmes: par exemple sur la question des adaptateurs primaires, ou des liens étroits existant entre deux modes de communication cellule-cellule, par contact direct ou par ligand soluble (parfois sécrété localement, voire immobilisé rapidement). Mais il faut se garder de plaquer un modèle sur l'autre et ne pas oublier que, dans des types cellulaires différents, une même molécule peut servir à des fonctions bien distinctes. II faut également garder à l'esprit que les « espacetemps » des deux systèmes sont bien différents: une synapse nerveuse peut fonctionner au même endroit pendant des mois ou des années, alors qu'un lymphocyte se déplace en permanence dans l'organisme, établissant des liaisons transitoires avec de nombreuses cellules différentes dans des laps de temps beaucoup plus courts. La brièveté de ces liaisons n'empêche pas que se structurent des synapses assurant un transfert d'informations pendant plusieurs minutes, voire plusieurs heures, qui permet une signalisation durable. $\diamond$

\section{REMERCIEMENTS}

Nous remercions Antoine Triller (École Normale Supérieure, Paris) pour ses suggestions et pour le cliché de la synapse N, Graça Raposo (Institut Curie, Paris) et Cédric Brossard (Institut Cochin) pour le cliché de la synapse T. L'équipe est financée par des crédits du Cnrs et par une labellisation de la Ligue Nationale Contre le Cancer.

\section{SUMMARY}

Immunological synapses and neuronal synapses

The interface between two cells from the immune system has recently been coined «immunological synapse». The authors review recent findings concerning the structure of the synapse formed betweeen T lymphocytes and antigenpresenting cells. T cells can be part of different synapses, depending on the antigen-presenting cell (B cell hybridoma, proteo-lipid bilayer, macrophage, dendritic cell). The synapse formed with dendritic cells is discussed in more details. A comparison is made with the synapses from the nervous system. Several parallel questions are discussed: how receptors can be clustered, what is the influence of synapse functioning on the structure of the synapse. It is suggested that in both cases two modes of communication exist in parallel: direct cell-cell contacts and soluble mediators, neurotransmitters in one case, putative immunotransmitters in the other. $\Delta$

$\begin{array}{lll}(\rightarrow) m / s & (\rightarrow) m / s & (\rightarrow) m / s \\ 1998, n^{\circ} 2, & 1994, n^{\circ} 10, & 1996, n^{\circ} 8-9, \\ \text { p. } 230 & \text { p. } 1042 & \text { p. } 1003\end{array}$

\section{AGRINE عT AGRÉGATION DE RÉCEPTEURS}

L'agrine est une molécule sécrétée par les terminaisons nerveuses des motoneurones qui reste piégée dans le réseau collagénique constituant la lame basale de la jonction neuro-musculaire $(\rightarrow)$. La protéine immobilisée interagit avec les récepteurs de la membrane plasmique des cellules musculaires comme les complexes de dystroglycane $(\rightarrow)$ [51], et avec Musk, un récepteur transmembranaire à activité tyrosine kinase [52]. Ainsi, l'action agrégeante qu'exerce l'agrine sur les récepteurs de l'acétylcholine n'est pas directe; elle agit plutôt comme un ligand immobilisé stimulant notamment l'activité d'agrégation de Musk $(\rightarrow)$. Au niveau des synapses du système nerveux central, l'agrine est présente, mais sa fonction reste obscure. On trouve aussi de l'agrine associée aux lymphocytes. II a été suggéré que l'agrine jouerait un rôle important dans la formation de la synapse T [53]. Elle permettrait la réorganisation de micro-domaines de la membrane plasmique riches en cholestérol et en sphingolipides, les rafts ou radeaux, qui contiennent de nombreux récepteurs et molécules de signalisation. L'accumulation de ces rafts à l'interface lymphocytes T-APC permettrait d'abaisser le seuil d'activation des lymphocytes T [53]. Une analyse précise des résultats ne permet cependant pas selon nous d'établir l'importance de l'agrine dans la formation de la synapse T. Certes, l'agrine est un élément de plus partagé par les systèmes nerveux et immunitaire. En tirer argument pour proposer des similitudes fonctionnelles spécifiques aux deux systèmes paraît superficiel. Nous préférons nous en tenir aux faits, et rappeler que le « bricolage moléculaire » de l'évolution aboutit souvent à l'utilisation d'une même molécule par différents types cellulaires, parfois avec des fonctions bien distinctes. 


\section{ADAPTATEURS SECONDAIRES DE LA SYNAPSE T}

Vav est l'adaptateur le plus étudié dans les lymphocytes $\mathrm{T}(\rightarrow)$. C'est un adaptateur secondaire nécessaire aux interactions entre les TCR et le cytosquelette mais qui n'interagit pas directement avec eux. II agit en fait comme facteur d'échange des GTPases Rho $(\rightarrow)$ et conditionne l'accumulation des complexes TCR-CMH à la synapse T [14].

Récemment, le rôle des molécules ERM (ezrine, radixine, moésine) dans la construction de la synapse T a été mis en évidence. Ces molécules possèdent un domaine de liaison avec l'actine et un domaine de liaison avec certaines protéines comme les ICAM ou CD43. Les protéines ERM participeraient à l'exclusion de grandes molécules comme CD43 de la synapse T $[10,55,56](\rightarrow)$, sans doute par un mécanisme de « réchauffement-congélation »: CD43 se décrocherait de la moésine, diffuserait dans la membrane, hors de la synapse, avant de se réassocier à la moésine extrasynaptique, qui maintiendrait ainsi sa localisation exclue de la synapse [2].

\section{RÉFÉRENCES}

1. Grakoui A, Bromley SK, Sumen C, et al. The immunological synapse: a molecular machine controlling T cell activation. Science 1999; 285: 221-7.

2. Delon J, Stoll S, Germain RN. Imaging of T-cell interactions with antigen presenting cells in culture and in intact lymphoid tissue. Immunol Rev 2002; 189: 51-63.

3. Dustin ML, Cooper JA. The immunological synapse and the actin cytoskeleton: molecular hardware for T cell signaling. Nat Immunol $2000 ; 1: 23-9$

4. Van der Merwe AP, Davis SJ, Shaw AS, Dustin ML. Cytoskeletal polarization and redistribution of cellsurface molecules during $T$ cell antigen recognition. Semin Immunol 2000; 12:5-21.

5. Monks $C R$, Freiberg $B A$, Kupfer $\mathrm{H}$, Sciaky N, Kupfer A. Three-dimensional segregation of supramolecular activation clusters in T cells. Nature 1998; 395: 82-6.

6. Freiberg BA, Kupfer $\mathrm{H}$, Maslanik W, et al. Staging and resetting $T$ cell activation in SMACs. Nat Immunol 2002; 3: 911-7.

7. Monks CR, Kupfer H, Tamir I, Barlow A, Kupfer A. Selective modulation of protein kinase C $-\theta$ during T-cell activation. Nature 1997; 385: 83-6.

8. Sun Z, Arendt CW, Ellmeier W, et al. PKC $-\theta$ is required for TCR-induced NF- KB activation in mature but not immature T lymphocytes. Nature 2000; 404: 402-7.

9. Revy P, Sospedra M, Barbour B, Trautmann A. Functional antigen-independent synapses formed between $T$ cells and dendritic cells. Nat Immunol 2001; 2: 925-31.

10. Allenspach $\varepsilon$ J, Cullinan $P$, Tong J, et al. ERM-dependent movement of CD43 defines a novel protein complex distal to the immunological synapse. Immunity 2001; 15: 739-50.

11. Krummel MF, Sjaastad MD,

\section{$(\rightarrow) \mathrm{m} / \mathrm{s}$ \\ 2003, n' 2 , \\ p. 217 \\ $\rightarrow$ m/s \\ $2002, n^{\circ} 5$, \\ p. 540}

\section{$(\rightarrow) \mathrm{m} / \mathrm{s}$ \\ 2003, $n^{\circ} 3$,}

p. 358
LES PROTÉINES « PDZ »

Des protéines à domaine PDZ (PSD95/Discs large/ZO-1) sont impliquées dans de très nombreux types d'interactions cellule-cellule. Certaines de ces protéines appartiennent à la famille des MAGUK (membraneassociated guanylate kinase). Les protéines CASK et PSD-95 en sont des exemples. CASK interagit avec les $\beta$-neurexines dans la terminaison pré-synaptique et contribue au recrutement de l'appareil sécrétoire présynaptique [30]. PSD95 est présente dans les « densités post-synaptiques » (PSD ou disk-shaped organelles) de $30 \mathrm{~nm}$ d'épaisseur attachées à la membrane post-synaptique des synapses excitatrices principalement, et crée une structure capable d'interagir avec les neuroligines, certains récepteurs de neurotransmetteurs comme les récepteurs NMDA $(\rightarrow)$ et des complexes associés à la signalisation [54].
1994, n० 6-7

p. 722 et

$2000, n^{\circ} 3$, p. 444
Wulfing C, Davis MM. Differential clustering of CD4 and $\mathrm{CD} 3 \xi$ during T cell recognition. Science 2000; 289: 1349-52.

12. Wulfing C, Sjaastad MD, Davis MM. Visualizing the dynamics of $T$ cell activation: intracellular adhesion molecule 1 migrates rapidly to the $T$ cell/B cell interface and acts to sustain calcium levels. Proc Natl Acad Sci USA 1998; 95: 6302-7.

13. Richie LI, Ebert PJR, Wu LC, Krummel MF, Owen JJT, Davis M. Imaging synapse formation during thymocyte selection: inability of $\mathrm{CD} 3 \zeta$ to form a stable central accumulation during negative selection. Immunity 2002; 16: 595-606.

14. Wulfing $C$, Bauch $A$, Crabtree $G R$, Davis MM. The vav exchange factor is an essential regulator in actindependent receptor translocation to the lymphocyte-antigen- presenting cell interface. Proc Natl Acad Sci USA 2000; 97: 10150-5.

15. Kupiec JJ, Sonigo P. Ni Dieu ni gène. Paris: Seuil, 2000.

16. Banchereau J, Steinman RM. Dendritic cells and the control of immunity. Nature 1998; 392: 245-52.

17. Delon J, Bercovici N, Raposo G, Liblau R, Trautmann A. Antigen-dependent and independent $\mathrm{Ca}^{2+}$ responses triggered in $\mathrm{T}$ cells by dendritic cells compared with B cells. J Exp Med 1998. 188: 1473-84.

18. Montes M, Mcllroy D, Hosmalin A, Trautmann A. Calcium responses elicited in human T cells and dendritic cells by cell-cell interaction and soluble ligands. Int Immunol 1999; 11: 561-8.

19. Inaba K, Romani N, Steinman RM. An antigenindependent contact mechanism as an early step in T cell-proliferative responses to dendritic cells. J Exp Med 1989; 170: 527-42. 
20. Bousso P, Bhakta NR, Lewis RS, Robey $\varepsilon$. Dynamics of thymocyte-stromal cell interactions visualized by two-photon microscopy. Science 2002; 296: 1876-80.

21. Miller MJ, Wei SH, Parker I, Cahalan MD. Two-photon imaging of lymphocyte motility and antigen response in intact lymph node. Science 2002; 296 : 1869-73.

22. Lee $K H$, Holdorf $A D$, Dustin ML, Chan AC, Allen PM, Shaw AS. T cell receptor signaling precedes immunological synapse formation. Science 2002; 295: 1539-42.

23. Stefanova II, Dorfman JR, Germain RN. Selfrecognition promotes the foreign antigen sensitivity of naive T lymphocytes. Nature 2002 ; 420: 429-34.

24. Irvine DJ, Purbhoo MA, Krogsgaard M, Davis MM. Direct observation of ligand recognition by T cells. Nature 2002; 419: 845-9.

25. Costello PS, Gallagher M, Cantrell DA. Sustained and dynamic inositol lipid metabolism inside and outside the immunological synapse. Nat Immunol 2002 ; 3: 1082-9.

26. Harriague J, Bismuth $G$. Imaging antigen-induced PI3K activation in T cells. Nat Immunol 2002; 3: 1090-6.

27. Stinchcombe JC, Bossi G, Booth S, Griffiths GM. The immunological synapse of CTL contains a secretory domain and membrane bridges. Immunity 2001; 15: 75l-6l.

28. Kupfer H, Monks CR, Kupfer A. Small splenic B cells that bind to antigen-specific $T$ helper (Th) cells and face the site of cytokine production in the Th cells selectively proliferate: immunofluorescence microscopic studies of Th-B antigen-presenting cell interactions. J Exp Med 1994; 179: 1507-15.

29. Suzuki SC, Inoue T, Kimura Y, Tanaka T, Takeichi M. Neuronal circuits are subdivided by differential expression of type-II classic cadherins in postnatal mouse brains. Mol Cell Neurosci 1997; 9: 433-47.

30. Missler M, Sudhof TC. Neurexins: three genes and 1001 products. Trends Genet 1998; 14:20-6.

31. Huh GS, Boulanger LM, Du H, Riquelme PA, Brotz TM, Shatz C). Functional requirement for class I MHC in CNS development and plasticity. Science 2000; 290 : 2155-9.

32. Reichert $P$, Reinhardt $R L$, Ingulli $\varepsilon$, Jenkins MK. Cutting edge: in vivo identification of TCR redistribution and polarized IL-2 production by naive CD4 T cells. J Immunol 2001; 166: 4278-81.

33. Arrieumerlou C, Donnadieu $\varepsilon$, Keryer $G$, et al. Involvement of phosphoinositide 3-kinase and rac in membrane ruffling induced by IL-2 in T cells Eur J Immunol 1998; 28: 1877-85.

34. Donnadieu $\varepsilon$, Bismuth $G$, Trautmann A. The intracellular $\mathrm{Ca}^{2+}$ concentration optimal for $\mathrm{T}$ cell activation is quite different after ionomycin or CD3 stimulation. EurJ Physiol (Pflug Arch) 1995; 429: 546-54.

35. Feng G, Tintrup H, Kirsch J, et al. Dual requirement for gephyrin in glycine receptor clustering and molybdoenzyme activity. Science 1998; 282: 1321-4.

36. Meier J, Meunier-Durmort $C$, Forest C, Triller A, Vannier C. Formation of glycine receptor clusters and their accumulation at synapses. J Cell Sci 2000; 113: 2783-95.

37. Apel ED, Roberds SL, Campbell KP, Merlie JP. Rapsyn may function as a link between the acetylcholine receptor and the agrin-binding dystrophin-associated glycoprotein complex. Neuron 1995; 15: 115-26.

38. Rao A, Craig AM. Activity regulates the synaptic localization of the NMDA receptor in hippocampal neurons. Neuron 1997; 19 . 801-12.

39. Dustin ML, Olszowy MW,
Holdorf AD, et al. A novel adaptor protein orchestrates receptor patterning and cytoskeletal polarity in Tcell contacts. Cell 1998; 94 : 667-77.

40. Griffiths \&K, Krawczyk C, Kong yy, et al. Positive regulation of $T$ cell activation and integrin adhesion by the adapter Fyb/Slap. Science 2001; 293 2260-3.

41. Peterson $\varepsilon$ J, Woods ML, Dmowski SA, et al. Coupling of the TCR to integrin activation by Slap-130/Fyb. Science 2001; 293: 2263-5.

42. Hanada T, Lin L, Tibaldi $\varepsilon V$, Reinherz $\varepsilon L$, Chishti AH. GAKIN, a novel kinesin-like protein associates with the human homologue of the Drosophila discs large tumor suppressor in T lymphocytes. J Biol Chem 2000; 275 : 28774-84.

43. Itoh K, Sakakibara M, Yamasaki $S$, et al. Cutting edge: negative regulation of immune synapse formation by anchoring lipid raft to cytoskeleton through $\mathrm{Cbp}$ EBP50-ERM assembly. J Immunol 2002; 168: 541-4.

44. Fischer M, Kaech S, Knutti D, Matus A. Rapid actin-based plasticity in dendritic spines Neuron 1998; 20 : 847-54.

45. Hall ZW, Sanes JR. Synaptic structure and development: the neuromuscular junction. Cell 1993; 72 (suppl) : 99-121.

46. Engert $F$, Bonhoeffer $T$. Dendritic spine changes associated with hippocampal long-term synaptic plasticity. Nature 1999; 399: 66-70.

47. Koh YH, Popova $\varepsilon$, Thomas $U$, Griffith LC, Budnik V. Regulation of DLG localization at synapses by CaMKII-dependent phosphorylation. Cell 1999; 98: 353-63.

48. Matus A. Actin-based plasticity in dendritic spines. Science 2000; 290: 754-8

49. Al-Alwan MM, Rowden G, Lee TD, West KA. Cutting edge: the dendritic cell cytoskeleton is critical for the formation of the immunological synapse. J Immunol 2001; 166: 1452-6.

50. Boes M, Cerny J, Massol R, et al. T-cell engagement of dendritic cells rapidly rearranges MHC class II transport. Nature 2002; 418: 983-8.

51. Gee SH, Montanaro F, Lindenbaum MH, Carbonetto S. Dystroglycan- $\alpha, a$ dystrophin-associated glycoprotein, is a functional agrin receptor. Cell 1994; 77: 675-86

52. Glass DJ, Bowen DC, Stitt TN, et al. Agrin acts via a MuSK receptor complex. Cell 1996; 85: 513-23.

53. Khan AA, Bose C, Yam LS, Soloski MJ, Rupp F. Physiological regulation of the immunological synapse by agrin. Science 2001 292: 1681-6.

54. Migaud $M$, Charlesworth $P$, Dempster $M$, et al. Enhanced long-term potentiation and impaired learning in mice with mutant postsynaptic density-95 protein. Nature 1998; 396: 433-9.

55. Delon J, Kaibuchi K, Germain RN. Exclusion of CD43 from the immunological synapse is mediated by phosphorylation-regulated relocation of the cytoskeletal adaptor moesin. Immunity 2001; 15: 691-701.

56. Roumier A, Olivo-Marin JC, Arpin $M$, et al. The membrane-microfilament linker ezrin is involved in the formation of the immunological synapse and in T cell activation. Immunity 2001; 15: 715-28.

TIRÉS À PART

A. Trautmann 